\title{
Erratum to: Nucleon spin-polarisabilities from polarisation observables in low-energy deuteron Compton scattering
}

\author{
H.W. Grießhammer ${ }^{1, a}$ and D. Shukla ${ }^{2}$ \\ 1 Institute for Nuclear Studies, Department of Physics, George Washington University, Washington, DC 20052, USA \\ 2 Department of Physics \& Astronomy, James Madison University, Harrisonburg, VA 22807, USA
}

Original article: Eur. Phys. J. A (2010) 46: 249, DOI: 10.1140/epja/i2010-11037-6

Received: 28 March 2012

Published online: 29 May 2012 - (C) Società Italiana di Fisica / Springer-Verlag 2012

We correct three errors in the original publication.

First, eq. (21) on p. 255 describing the implementation of varying the individual polarisabilities contains an incorrect kinematic pre-factor $\sqrt{s_{\gamma N}} / M$. This should be deleted, as should the references to it in the text immediately following. The numerical implementation does not contain this factor and thus remains unchanged.

Second, the original version implies that the sensitivity of observables is analysed by varying the iso-scalar values of the scalar and spin-polarisabilities by an iso-scalar amount of \pm 2 canonical units. This is not the case. Instead, setting $\delta \alpha_{E 1}, \delta \beta_{M 1}, \delta \gamma_{E 1 E 1}, \delta \gamma_{M 1 E 2}, \delta \gamma_{M 1 M 1}, \delta \gamma_{E 1 M 2}$ to \pm 2 units varies the polarisabilities of only one of the nucleons by \pm 2 units, while that of the other nucleon is kept at the iso-scalar value.

Two paragraphs after eq. (21) (starting at bottom of left column on p. 255), the first sentence is thus replaced by:

In the next step, these contributions are independently varied by \pm 2 canonical units for one nucleon to analyse the effect of each on the various observables. The polarisabilities of the other nucleon are kept fixed at the iso-scalar value. This corresponds to a change of the iso-scalar polarisabilities by half as much, i.e. by \pm 1 unit. Since deuteron Compton scattering is sensitive only to iso-scalar quantities, varying either the proton or neutron polarisabilities leads to the same result. In practise, the scalar polarisabilities of the proton are better constrained, and deuteron Compton scattering experiments are more likely focused on extracting neutron polarisabilities. In that case, these studies can be interpreted as providing the sensitivities on varying the neutron polarisabilities by \pm 2 units, with fixed proton polarisabilities. The spin-independent polarisabilities...

Third, the variation of the spin-polarisability $\gamma_{E 1 E 1}$ was implemented with an incorrect sign. The correct results are obtained by reinterpreting the plots only of $\delta \gamma_{E 1 E 1}$ in figs. $6,11,13,16,18,20,22$ : Dotted lines represent a change by +2 units, dashed ones by -2 units.

Except for the last modification, the figures and conclusions remain unchanged. The corresponding mathematica notebook, available from hgrie@gwu.edu, has been adjusted. It now reflects variations of the iso-scalar polarisabilities by \pm 2 units (i.e. of one individual nucleon polarisability by \pm 4 units, with those of the other kept fixed), thus complementing the article's perspective.

\footnotetext{
a e-mail: hgrie@gwu.edu
} 